\title{
DRAWING PLANAR GRAPHS WITH PRESCRIBED FACE AREAS*
}

\author{
Linda Kleist**
}

ABstraCT. We study straight-line drawings of planar graphs where every inner face has a prescribed area. A plane graph is area-universal if for every assignment of positive real numbers to the inner faces, there exists a straight-line drawing in which the area of each inner face equals to the assigned number. The only non-area-universal graphs known so far are those containing the octahedron graph as a subgraph.

We give a simple counting argument that allows us to prove non-area-universality for a large class of triangulations, namely Eulerian triangulations. Adding some geometric arguments, the concept can be used to show non-area-universality for other graphs such as the icosahedron graph.

Relaxing the straight-line property by allowing the edges to bend, we show that one bend per edge is sufficient to realize any face area assignment of every plane graph, i.e., the 1-subdivision of every plane graph is area-universal. For the subclass of plane bipartite graphs, it even suffices that half of the edges have a bend.

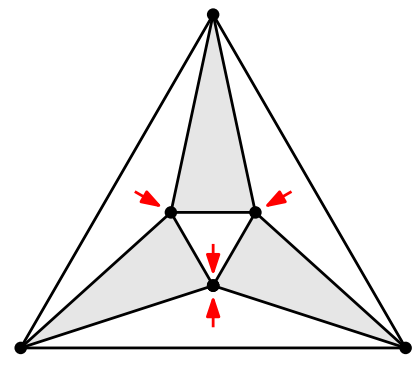

not area-universal

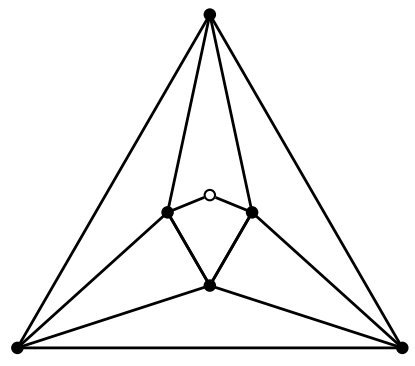

area-universal

\section{Introduction}

Various real-life problems are connected to embedded graphs on the surface of our planet. Therefore, planar graphs and their representations have many practical applications, e.g., in the manufacture of chips and electrical circuits, as well as in the design of network infrastructure such as roads, subways, and utility lines. Other applications are in cartography, geography, and visualization. Consequently, there is a large body of theoretical and applied work on representations of planar graphs with special features [1, 6, 17, 21, 25].

\footnotetext{
${ }^{*}$ A preliminary version appeared in the proceedings of WG 2016 [18].

** Technische Universität Berlin, kleist@math.tu-berlin.de
} 
One line of research deals with the representation of planar graphs with prescribed face areas and is inspired by cartograms. A cartogram is a map in which regions (countries, districts, ...) are scaled according to some statistical parameter such as the population or the gross national product. As a mathematical abstraction, proportional contact representations of planar graphs have been studied. In this context, vertices are represented by interiorly disjoint simple polygons with prescribed areas and adjacencies are represented by contacts between the corresponding polygons [3, 12, 13].

As an alternative abstraction, we are interested in drawings of plane graphs such that the inner faces have prescribed areas. To describe our concepts, we introduce some notation. Let $G=(V, E)$ be a plane graph, i.e, a graph with a fixed planar drawing. We consider two planar drawings of $G$ as equivalent if the outer face and the rotation systems coincide in both drawings, i.e., for every vertex the cyclic ordering of its incident edges coincides in both drawings. We denote the set of all straight-line drawings equivalent to $G$ by $\mathcal{D}$ and the set of the inner faces of $G$ by $F^{\prime}$. An area assignment is a function $\mathcal{A}: F^{\prime} \rightarrow \mathbb{R}_{>0}$. Let area $: F^{\prime} \times \mathcal{D} \rightarrow \mathbb{R}$ be the function measuring the area of an inner face $f \in F^{\prime}$ in a specific drawing $D$ of $G$. A drawing $D$ in $\mathcal{D}$ is $\mathcal{A}$-realizing if area $(f, D)=\mathcal{A}(f)$ for each face $f \in F^{\prime}$; an area assignment $\mathcal{A}$ is realizable if there exists an $\mathcal{A}$-realizing drawing. A plane graph $G$ is area-universal if every area assignment of $G$ is realizable. We say that $G$ is equiareal if there exists a drawing $D$ in $\mathcal{D}$ such that $\mathfrak{a r e a}(f, D)=1$ for each face $f \in F^{\prime}$.

\subsection{Previous Work \& Our Contribution}

Ringel [23] can be seen as the initiator of the study of drawings of planar graphs with prescribed face areas. He gave examples of equiareal graphs and proved that not every plane graph is equiareal. Moreover, Ringel conjectured that every plane 3-regular graph is equiareal. This turned out to be true when Thomassen [26] proved something stronger: Every plane graph with maximum degree 3 is area-universal. There are further area-universal graph classes. Biedl \& Ruiz Velázquez [8] showed that planar partial 3-trees are area-universal and have realizing drawings with rational coordinates if the area assignment is rational. Moreover, it is straight-forward to observe that the subgraphs of an area-universal graph are also area-universal. Dobbins et al. $[11,20]$ study the computational complexity of areauniversality. They conjecture that deciding area-universality is complete for the complexity class $\forall \exists \mathbb{R}$, a natural generalization of $\exists \mathbb{R}$. They support the conjecture by showing $\forall \exists \mathbb{R}$ completeness for several variants of area-universality. For triangulations with a certain vertex order, Kleist [19] presents a sufficient criterion for area-universality that only requires the investigation of one area assignment. In fact, if the sufficient criterion applies to one plane triangulation, then all embeddings of the corresponding planar graph are also areauniversal. It is open whether area-universality is a property of plane or planar graphs.

In this paper, we give negative and positive results. Surprisingly, the octahedron and its supergraphs were the only graphs in literature which are proved to be not area-universal. ${ }^{1}$ In terms of negative results, we were wondering whether a graph is area-universal if and only

\footnotetext{
${ }^{1}$ By now, our attention has been drawn to the bachelor thesis of Bernáthová [7] which indicates that some other small triangulations are also not area-universal. Based on the methods developed in this paper, non-area-univerality of small triangulations with up to ten vertices has been proved in [16, 19].
} 
if it does not contain the octahedron graph as a subgraph. Since a random triangulation includes with high probability any fixed planar subgraph [22], in particular the non-areauniversal octahedron, it follows that almost no triangulation is area-universal. However, a 4-connected graph does not contain any (other) triangulation except for $K_{3}$ as a subgraph. Therefore, we investigate the relationship of high connectivity and area-universality, specifically whether highly connected graphs are area-universal. The answers to these questions are negative. In Section 2, we give a broad class of non-area-universal triangulations, namely Eulerian triangulations. This class contains many 4-connected graphs. Additionally, we prove that the 5-connected icosahedron graph is not area-universal. Consequently, high connectivity does not imply area-universality.

In Section 3, we investigate polyline drawings as natural relaxations of straight-line drawings. We show that every plane graph has a drawing realizing any area assignment, such that each edge has at most one bend. In other words, the 1-subdivision of every plane graph is area-universal. For plane bipartite graphs we improve this by showing the existence of an area-universal subdivision where at most half of the edges are subdivided exactly once.

\section{Non-Area-Universal Graphs}

In this section, we discuss non-area-universality. We mentioned before that the octahedron graph, which is an Eulerian triangulation, is not area-universal. Two proofs of this fact are known already; both of which use a similar non-realizable area assignment. Ringel [23] shows that a system of polynomial equations, expressing the prescribed areas of each face, has no real solution. Since this is a necessary condition for each realizing drawing, the nonexistence of a real solution proves the non-realizability of the specific area assignment. A different proof relies on a classical geometric result on the area of a triangle inscribed into a triangle: Choosing one point on each side of a triangle and connecting all pairs of these three points by segments, one obtains a triangle partitioned into four triangles. Then the central triangle does not have the strictly smallest area among the four triangles [4, 24]. Note that the inner vertices of the octahedron are forced to lie on the outer edges when the area of the three triangles incident to the outer edges is supposed to vanish. In this way, we obtain a triangle inscribed into a triangle. The idea of this argument has been used by Alam et al. [2].

In contrast, we present a simple counting argument in order to show that the octahedron graph is not area-universal. Interestingly, this argument can be extended to prove that no plane Eulerian triangulation is area-universal. Recall that a triangulation is Eulerian if and only if all vertex degrees are even. We will use the fact that the dual graph of a plane Eulerian triangulation is bipartite, and hence has an inner face 2-coloring, i.e., a coloring of the inner faces with white and black such that every inner edge is incident to a white and black face. Figure 1 (a) depicts an inner face 2-coloring for the octahedron graph. Indeed, Eulerian triangulations are characterized by the existence of an inner face 2-coloring.

- Proposition 1. A triangulation has an inner face 2-coloring if and only if it is Eulerian.

Proof. It remains to show that every triangulation with an inner face 2-coloring is Eulerian. Suppose there is a plane triangulation $T$ with an inner face 2-coloring that is not Eulerian. 
Clearly, any inner vertex of $T$ has even degree and the number of vertices with odd degree is even. Hence, $T$ has exactly two outer vertices $u$ and $v$ of odd degree. Deleting the edge $(u, v)$ results in an Eulerian near-triangulation with $3 n-7$ edges. However, by Tsai and West [27], the number of edges of an Eulerian near-triangulation is divisible by three.

In order to prove Theorem 1, we make use of several observations. The first observation follows directly from the fact that triangles are affine equivalent. Hence, any realizing drawing of a triangulation can be transformed by an affine linear transformation. If the determinant of the associated matrix is 1 , the areas are maintained. For an area assignment $\mathcal{A}$, let the total area $\Sigma \mathcal{A}$ of $\mathcal{A}$ be the sum of all assigned areas, i.e., $\Sigma \mathcal{A}:=\sum_{f \in F^{\prime}} \mathcal{A}(f)$.

- Observation 2. Let $T$ be a plane triangulation with a realizable area assignment $\mathcal{A}$. Then there exists an $\mathcal{A}$-realizing drawing within every given outer face of area $\Sigma \mathcal{A}$.

In a planar drawing, no two edges intersect except in common vertices. Here, we are slightly more general and allow all drawings that can be obtained as the limit of a sequence of planar drawings. If a vertex lies on a non-incident edge or even on another vertex, we speak of a degenerate drawing. In particular, face areas of 0 can only be realized in degenerate drawings. Indeed, a straight-line drawing of a plane triangulation is degenerate if and only if the area of some triangle vanishes. A straight-line drawing of a graph with $n$ vertices can be encoded by the $2 n$ coordinates of the vertices, and hence, by a point in the Euclidean space $\mathbb{R}^{2 n}$. Consequently, we may think of a straight-line drawing as a vector in $\mathbb{R}^{2 n}$. For a plane graph $G, \mathcal{D}^{+}(G) \subseteq \mathbb{R}^{2 n}$ denotes the set of vectors representing equivalent and possibly degenerate straight-line drawings of $G$. By $\mathcal{D}_{\triangle}^{+}(G)$, we denote the subset of drawings of $\mathcal{D}^{+}(G)$ in which the outer face is restricted to a fixed triangle $\triangle$.

- Observation 3. For a plane triangulation $T, \mathcal{D}^{+}(T)$ is closed and $\mathcal{D}_{\triangle}^{+}(T)$ is compact.

With the help of this observation, we obtain a very handy lemma. Recall that an area assignment assigns positive real numbers to the inner faces. A non-negative area assignment is a function $\mathcal{A}: F^{\prime} \rightarrow \mathbb{R}_{\geq 0}$, i.e., every inner face is assigned to a non-negative real number.

- Lemma 4. Let $T$ be a plane triangulation, and $\mathbb{A}$ the set of non-negative area assignments with total area 1. Then $\mathcal{A} \in \mathbb{A}$ is realizable if and only if in every open neighborhood of $\mathcal{A}$ in $\mathbb{A}$ there exists a realizable area assignment.

Proof. Suppose $\mathcal{A} \in \mathbb{A}$ is realizable, then clearly $\mathcal{A}$ itself is in every of its open neighborhoods and serves as a certificate of a realizable area assignment.

Suppose in every open neighborhood of $\mathcal{A}$, there exists a realizing drawing. Hence, we may construct a sequence of realizable assignments $\left(\mathcal{A}_{i}\right)_{i \in \mathbb{N}}$ converging to $\mathcal{A}$. For each $\mathcal{A}_{i}$, we pick a realizing drawing $D_{i}$. By Observation 2, we may assume that the outer face of each drawing $D_{i}$ is a fixed triangle $\triangle$. Consequently, the sequence $\left(D_{i}\right)_{i \in \mathbb{N}}$ is contained in $\mathcal{D}_{\triangle}^{+}(T)$ and, thus, bounded by Observation 3. Therefore, by the Bolzano-Weierstrass theorem, the sequence $\left(D_{i}\right)_{i \in \mathbb{N}}$ contains a converging subsequence with limit $D$. By the closedness, $D$ is contained in $\mathcal{D}_{\triangle}^{+}(T)$. By construction of $D$ and the continuity of area, for every inner face $f$ it holds that:

$$
\mathfrak{a r e a}(f, D)=\lim _{i \rightarrow \infty} \mathfrak{a r e a}\left(f, D_{i}\right)=\lim _{i \rightarrow \infty} \mathcal{A}_{i}(f)=\mathcal{A}(f) .
$$

Consequently, the drawing $D$ is $\mathcal{A}$-realizing and $\mathcal{A}$ is realizable. 
Lemma 4 implies immediately that every non-realizable area assignment has an open neighborhood of non-realizable area assignments. This gives the following property.

Corollary 5. If a triangulation has a non-negative area assignment that is not realizable, then it also has a (positive) non-realizable area assignment.

Now we are ready to prove the theorem.

Theorem 1. No plane Eulerian triangulation on $n \geq 4$ vertices is area-universal.

Proof. Let $T$ be a plane Eulerian triangulation on at least 4 vertices with an inner face 2-coloring. For an example consider Figure 1 (a). We denote the set of white faces by $W$ and the set of black faces by $B$. Recall that a triangulation with $n$ vertices has $2 n-5$ inner faces. Without loss of generality we assume that $|W|>|B|$ and therefore $|W| \geq n-2$. We show that the following non-negative area assignment $\mathcal{A}$ of $T$ is not realizable.

$$
\mathcal{A}(f):= \begin{cases}0 & \text { if } f \in W \\ 1 & \text { if } f \in B .\end{cases}
$$

For the purpose of contradiction, suppose that there exists an $\mathcal{A}$-realizing drawing $D$ of $T$. We show that $D$ has two contradicting properties.

Firstly, every white face has an angle of size $\pi$; we call such an angle a flat angle: Note that every inner edge is incident to a black face and thus has positive length in $D$; otherwise, the area of the black face is not realized. Moreover, the area of a white face vanishes only if one of its vertices lie on the opposite, non-incident edge. Due to the positive edge lengths, the vertex lies in the interior of the non-incident edge. Consequently, one angle of every white face in $D$ is flat.

Secondly, the number of white flat angles is bounded by the number of inner vertices: In $D$, we assign (the flat angle of) every white face to the vertex with the flat angle and

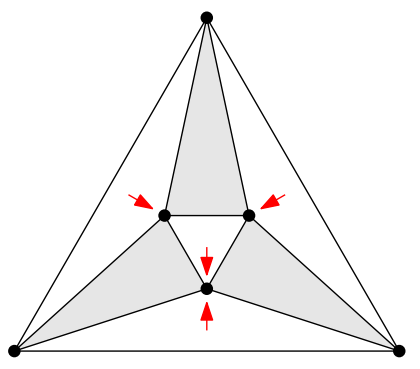

(a) In every flat angle assignment of $\mathcal{A}$, two white faces are assigned to the same inner vertex. This fact is used to prove Theorem 1.

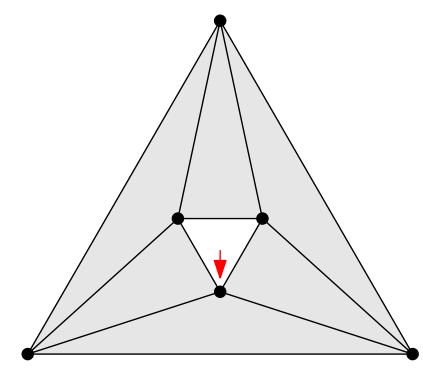

(b) A non-realizable area assignment based on Lemma 7. Every flat angle assignment yields a base-bounded seesaw.

Figure 1: Inner face colorings of the octahedron graph depicting non-realizable area assignments; white faces are assigned to 0 and black faces to 1 . 
obtain a flat angle assignment. Since the boundary of the outer face is a triangle with positive area, only inner vertices may have flat angles. Here we use the fact that $n>3$. Moreover, every inner vertex has degree at least 4 and is incident to a black face. Suppose there exists an inner vertex $v$ in $D$ which has two flat angles. Then, no space remains to realize the area of any black face incident to $v$. Therefore, in $D$ every inner vertex has at most one flat angle and the number of white flat angles is bounded by the number of inner vertices.

Recall that $T$ has at least $n-2$ white faces and $n-3$ inner vertices. Thus, the number of white faces exceeds the number of inner vertices and we obtain a contradiction. Therefore, $\mathcal{A}$ is not realizable and Corollary 5 guarantees the existence of a non-realizable (positive) area assignment.

Remark 1. Note that in the proof of Theorem 1, we only used the fact that the black faces are assigned to positive real numbers. Consequently, any non-negative area assignment of an Eulerian triangulation with an inner face 2-coloring where each white face is assigned to 0 and each black face to some positive real number is not realizable.

The proof of Theorem 1 implies that one cannot hope for drawings realizing the areas up to a constant factor. For a real number $c>1$, we say a straight-line drawing $D$ of $G$ is c-approximating an area assignment $\mathcal{A}$ if for each inner face $f$ it holds that

$$
1 / c \cdot \mathcal{A}(f) \leq \mathfrak{a r e a}(f, D) \leq c \cdot \mathcal{A}(f) .
$$

- Proposition 6. For any $c>1$, every Eulerian triangulation $T$ on $n \geq 4$ vertices admits an area assignment $\mathcal{A}$ such that no drawing of $T$ is c-approximating $\mathcal{A}$.

Proof. We consider an inner face 2-coloring of $T$. For every $k \in \mathbb{N}$, let $\mathcal{A}_{k}$ denote the area assignment of $T$ where each white face is assigned to $1 / k$ and each black face to 1 . Suppose every $\mathcal{A}_{k}$ has a drawing $D_{k}$ which is $c$-approximating $\mathcal{A}_{k}$. We may assume that the outer face of $T$ in $D_{k}$ is a right triangle where two outer vertices coincide for all $D_{k}$. Then the sequence $\left(D_{k}\right)_{k \in \mathbb{N}}$ is bounded and by the Bolzano-Weierstrass theorem, it has a converging subsequence with limit $D$. In $D_{k}$, the area of a white face is at most $c / k$ and the area of a black face is at least $1 / c$. Consequently, in $D$, the area of each white face is 0 and the area of each black face is positive. However, by Remark 1, such a drawing of $T$ does not exist.

Since every Eulerian triangulation (with at least four vertices) has a vertex of degree four, the graphs of Theorem 1 are at most 4-connected. Adding some geometric arguments, we can use similar ideas to show that the 5-connected icosahedron graph is not area-universal. We start with the geometric argument.

Let $A B C D$ be a quadrangle, and let $E$ be a point on the segment $C D$. Together with the segments $A E$ and $B E$ we call this configuration a seesaw with base $A B$. A seesaw is basebounded if there exists a pair of parallel lines $\ell_{A}$ and $\ell_{B}$ through $A$ and $B$, respectively, such that the seesaw lies (not necessarily strictly) between the lines $\ell_{A}$ and $\ell_{B}$. For example, if the two lines are vertical then in a base-bounded seesaw with horizontal base, the $x$-coordinates of $C$ and $D$ are bounded by the $x$-coordinates of $A$ and $B$. Figure 2 (a) depicts a base bounded seesaw. We call a seesaw equiareal if the three triangles $A E D, A B E$, and $B C E$ have the same positive area. 


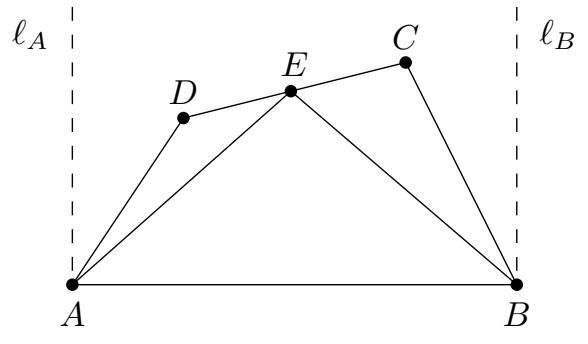

(a) A base-bounded seesaw.

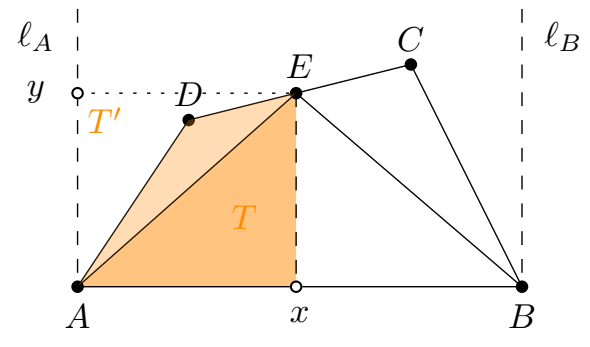

(b) $A E D$ is smaller than $A B E$.

Figure 2: Illustration of Lemma 7 and its proof.

- Lemma 7. No base-bounded seesaw is equiareal.

Proof. For a contradiction, suppose that there exists a base-bounded equiareal seesaw. Without loss of generality, we assume that $A B$ is horizontal and that $E$ lies above $A B$. Note that $E$ lies strictly between $\ell_{A}$ and $\ell_{B}$; otherwise the triangle $A E D$ or $B C E$ has area 0 . By symmetry, we may assume that $D$ does not lie above $C$ as depicted in Figure 2 (b). Let $x$ denote the intersection point of the segment $A B$ and the line parallel to $\ell_{A}$ through $E$; let $y$ be the intersection point of $\ell_{A}$ and the horizontal line through $E$. Since $E$ lies strictly between $\ell_{A}$ and $\ell_{B}, x$ lies strictly between $A$ and $B$. Therefore, the triangle $T:=A x E$ is strictly contained in $A B E$ and has the same area as $T^{\prime}:=A E y$. By assumption $D$ is not to the left of $\ell_{A}$ and not above $E$ and therefore $D$ is contained in $T^{\prime}$. Thus, it holds that

$$
\mathfrak{a r e a}(A B E)>\mathfrak{a r e a}(T)=\mathfrak{a r e a}\left(T^{\prime}\right) \geq \mathfrak{a r e a}(A E D) .
$$

This is a contradiction to the equiareality of the seesaw and therefore proves the claim.

Remark 2. Lemma 7 gives an alternative proof that the octahedron is not area-universal: Consider the area assignment where all inner faces have area 1 but the central triangle has area 0 , as depicted in Figure 1 (b). Then the flat angle of the central face must be assigned to an inner vertex. This yields a base-bounded seesaw which is not equiareal by Lemma 7. In fact, this even implies that no area assignment of the octahedron is realizable when each black face is assigned to 1, the central white face to 0, and every other white face to 0 or 1 .

Moreover, Lemma 7 enables us to show the following fact.

Theorem 2. The icosahedron graph is not area-universal.

Proof. We show that there exists no straight-line drawing of the icosahedron in which the white faces in Figure 3 vanish and the total area is distributed evenly among all black faces. Then Corollary 5 guarantees the existence of a non-realizable (positive) area assignment.

By contradiction, we suppose that there exists a realizing drawing $D$. Note that in the face colored icosahedron there exist three edges which are adjacent to two white faces; namely the red edges in Figure 3. For a white face incident to a red edge, there exists two possibilities in $D$ : either the red edge is contracted or one of its angles is flat. Thus, we distinguish four cases according to how many of the red edges are contracted. Specifically, 

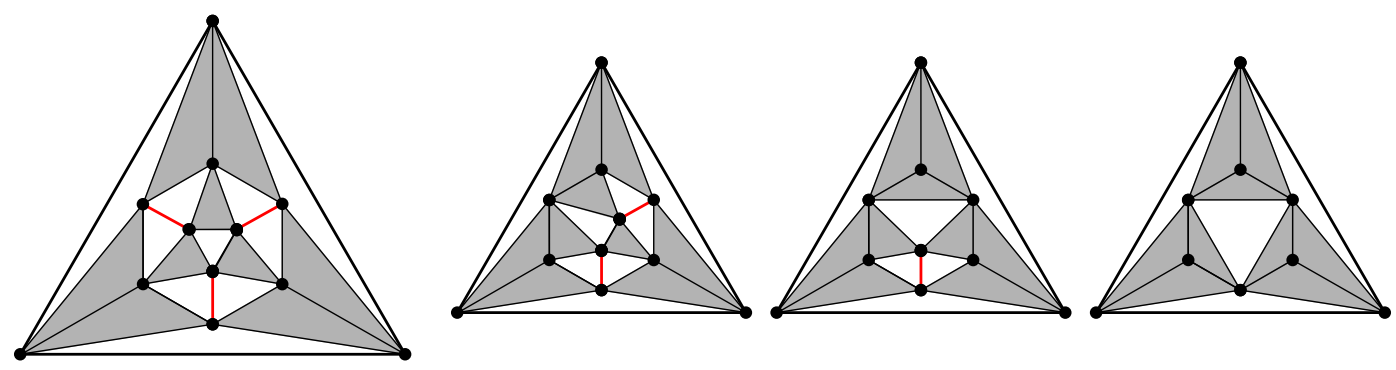

Figure 3: The icosahedron with a face coloring depicting a non-realizable area assignment; from left to right, no, one, two and three edges are contracted.

in case $i$, we contract exactly $i$ edges with $i \in\{0,1,2,3\}$. Then, the remaining white faces must have a flat angle. Moreover, every inner vertex is incident to a black face and thus may only be incident to at most one flat angle. Therefore, we say a flat angle assignment is valid if no two faces are assigned to the same vertex. In each case, we either find a base-bounded seesaw or no valid flat angle assignment.

Case 0: If no edge is contracted, then there are ten white faces and nine inner vertices. By the pigeonhole principle, there exists no valid flat angle assignment.

Case 1: The contraction of one edge results in a graph with eight white faces and eight inner vertices. Hence, in any valid flat angle assignment each inner vertex has exactly one flat angle. We label the vertices as depicted in Figure 4. In particular, $v_{1}, v_{3}, v_{4}, v_{5}, v_{6}$ are assigned by a unique white face. In total, there are two different flat angle assignments, where the vertices $v_{2}, v_{7}, v_{8}$ are either assigned in clockwise or counter clockwise direction. Since these two flat angle assignments are mirror images of each other, we consider without loss of generality the one displayed in Figure 4 (a).

To obtain a contradiction, we consider a realizing drawing $D$ within the triangle $A B C$ with vertices $(0,0),(1,0),(0,1)$ where the collinearities from the flat angle assignment are respected and all remaining faces have the same area. For an illustration of $D$, see Figure 4 (b).

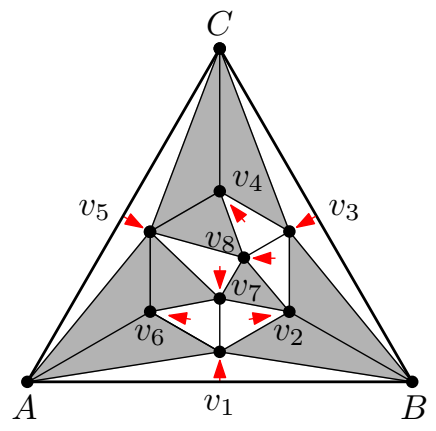

(a) The up to symmetry unique flat angle assignment in Case 1.

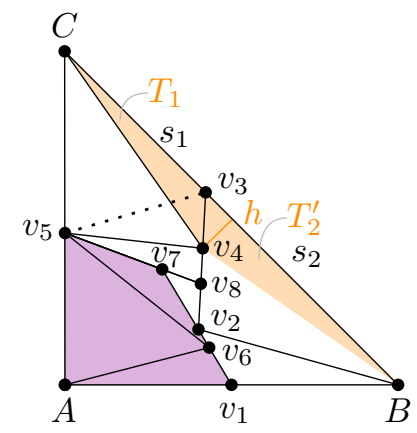

(b) A drawing respecting the flat angle assignment; it contains a seesaw with base $v_{5} A$.

Figure 4: Illustration of Case 1 of the proof of Theorem 2. 
For a vertex $v_{i}$, we denote its coordinates in $D$ by $\left(x_{i}, y_{i}\right)$. We will show that $D$ contains a base-bounded seesaw. Specifically, a straight-line drawing of the subgraph induced by the vertices $A, v_{1}, v_{6}, v_{7}, v_{5}$ yields a seesaw with base $v_{5} A$, as highlighted in violet in Figure 4 (b). If $y_{5} \geq y_{7}$, then a pair of horizontal lines certifies that the seesaw is base-bounded and Lemma 7 yields a contradiction. Therefore, it remains to show that $y_{5} \geq y_{7}$. By contradiction, we suppose that $y_{5}<y_{7}$.

First note that, since $v_{4}$ lies on the segment $v_{8} v_{3}$, the (artificial) segment $v_{5} v_{3}$ intersects segment $C v_{4}$. Moreover, $v_{5}$ and $v_{3}$ lie on the outer segments $A C$ and $B C$, respectively. Hence, all inner vertices are contained in the quadrangle $Q:=A B v_{3} v_{5}$. Since $y_{5}<y_{7}$ by assumption, it follows that $y_{5}<y_{3}$.

Next, we show that $y_{3}<1 / 2$. Consider the two black faces incident to $v_{3}$, namely $T_{1}:=v_{4} v_{3} C$ and $T_{2}:=v_{3} v_{2} B$. Due to the flat angle assignment, $v_{4}$ lies on the segment $v_{2} v_{3}$ and the triangle $T_{2}^{\prime}:=v_{3} v_{4} B$ is strictly contained in the triangle $T_{2}$. Consequently, it holds that $\mathfrak{a r e a}\left(T_{1}, D\right)=\mathfrak{a r e a}\left(T_{2}, D\right)>\mathfrak{a r e a}\left(T_{2}^{\prime}, D\right)$. When we consider the segments $s_{1}$ and $s_{2}$ on $B C$ as the base segments of $T_{1}$ and $T_{2}^{\prime}$, respectively, then their heights coincide. Therefore, $\mathfrak{a r e a}\left(T_{1}, D\right)>\mathfrak{a r e a}\left(T_{2}^{\prime}, D\right)$ implies directly that $s_{1}>s_{2}$, and hence, $y_{3}<1 / 2$.

Since the quadrangle $Q$ strictly contains seven facial triangles, it covers at least $7 / 9$ of the area in $D$. The area of $Q$ can be expressed as the sum of the triangles $A v_{3} v_{5}$ with base length $y_{5}$ and height $x_{3}$ and $A B v_{3}$ with base length 1 and height $y_{3}$. With the facts that $y_{5}<y_{3}<1 / 2$ and $x_{3}=1-y_{3}$, we obtain the following chain of inequalities:

$$
7 / 9 \leq 2 \cdot \mathfrak{a r e a}(Q)=y_{5} x_{3}+y_{3}<1 / 2 \cdot\left(1-y_{3}\right)+y_{3}=1 / 2 \cdot\left(1+y_{3}\right)<3 / 4 .
$$

This is a contradiction. Consequently, we have that $y_{5} \geq y_{7}$. This finishes Case 1 .

Case 2: The contraction of two edges results in a graph with a unique flat angle assignment which is shown in Figure 5 (a). Consider Figure 5 (b) to note that every straightline drawing respecting this flat angle assignment contains two seesaws. Due to its symmetry, we assume without loss of generality that $y_{5} \geq y_{3}$. Hence, $v_{6}$ induces a seesaw with base $A v_{5}$ in the quadrangle $A v_{1} v_{7} v_{5}$; the base-boundedness is certified by a pair of horizontal lines. By Lemma 7, the base-bounded seesaw is not equiareal and we obtain a contradiction.

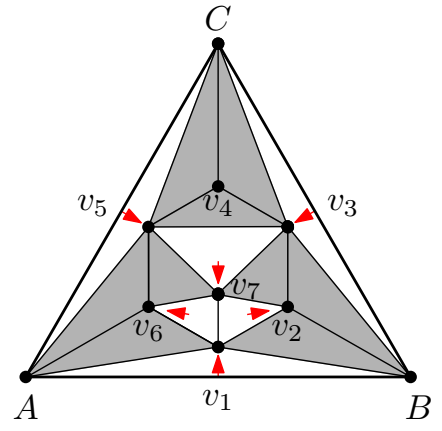

(a) The unique angle assignment when two edges are contracted.

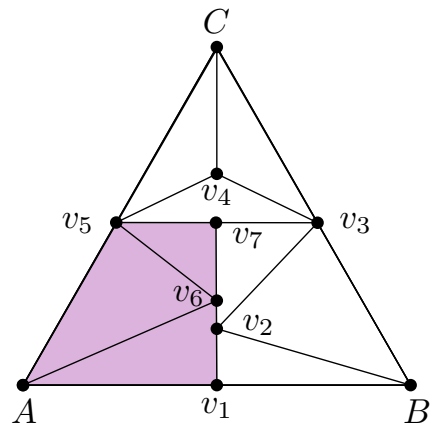

(b) A drawing respecting the flat angle assignment; it contains two seesaws.

Figure 5: Illustration of Case 2 of the proof of Theorem 2. 
Case 3: Contraction of three edges leads to a super graph of the octahedron graph as depicted in the rightmost image of Figure 3. This graph has four white faces and three inner vertices adjacent to white faces. Hence, there exists no valid flat angle assignment.

This finishes the section on non-area-universal graphs. We now turn our attention to realizing drawings.

\section{Drawing Planar Graphs with Bends}

We now aim for realizing each area assignment of every plane graph. As discussed in Section 2, this is impossible with straight-line drawings. Therefore, we relax the straightline property by allowing the edges to have bends. A drawing of a plane graph is a $k$-bend drawing if each edge is a concatenation of at most $k+1$ segments. These drawings are also known as polyline drawings. Interpreting the bend as a subdivision vertex, the polyline drawing corresponds to a straight-line drawing of a subdivision of the graph. In this sense, the notions of planar, degenerate and realizing drawings immediately translate to polyline drawings. In this section, we show that one bend per edge is sufficient.

Theorem 3. Let $G$ be a plane graph and $\mathcal{A}: F^{\prime} \rightarrow R_{>0}$ an area assignment of $G$. Then, there exists an equivalent 1-bend drawing of $G$ realizing $\mathcal{A}$.

Proof. Without loss of generality, we assume that $G$ is a plane triangulation: If $G$ is not a triangulation, there exists a triangulation $T$ such that $G$ is an induced subgraph. For each face of $G$, partition the assigned area between its subfaces in $T$ and obtain the area assignment $\mathcal{A}^{\prime}$ of $T$. Given an $\mathcal{A}^{\prime}$-realizing 1-bend drawing of $T$, delete the artificial vertices and edges. The result is an $\mathcal{A}$-realizing 1-bend drawing of $G$.

We construct the final drawing of $G$ in four steps (see definitions below). Figure 6 visualizes the steps for the octahedron graph.

1. Take a $\perp$-contact representation $\mathcal{C}$ which yields a rectangular layout $\mathcal{L}$.

2. Obtain a weak equivalent rectangular layout $\mathcal{L}^{\prime}$ realizing the areas.

3. Define a degenerate drawing $D_{\perp}$.

4. Construct a non-degenerate drawing $D_{\perp}^{*}$ from $D_{\perp}$.

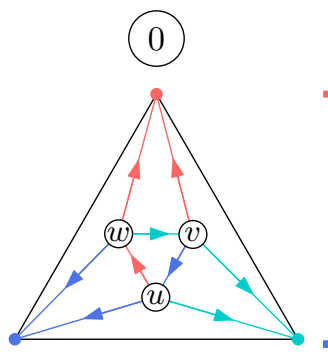

$G$ with a

Schnyder Wood

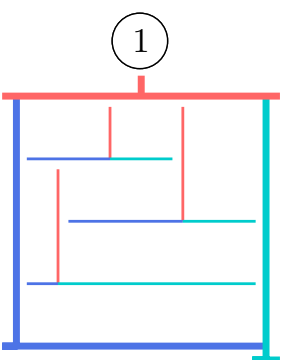

$\mathcal{C}$
(2)

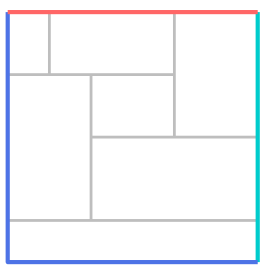

$\mathcal{L}^{\prime}$
(3)

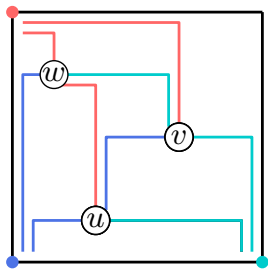

$\mathcal{D}_{\perp}$
(4)

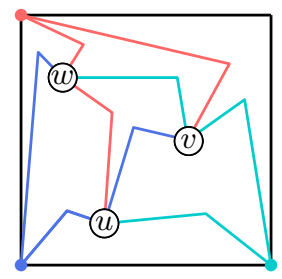

$\mathcal{D}_{\perp}^{*}$

Figure 6: Construction of a realizing 1-bend drawing in four steps. 
In the first step, we construct a $\perp$-contact representation $\mathcal{C}$ of $G$. A $\perp$-shape is the union of a horizontal and vertical segment such that the lower end of the vertical segment lies in the horizontal segment. We call this point of intersection the heart of the $\perp$-shape. Each of the other three ends of the segments is an end of the $\perp$-shape. A $\perp$-contact representation of a graph $G=(V, E)$ is a family of $\perp$-shapes $\left\{\perp_{v}: v \in V\right\}$ where $\perp_{u}$ and $\perp_{v}$ intersect if and only if $(u, v) \in E$. Moreover, if $\perp_{u}$ and $\perp_{v}$ intersect then the intersection must consist of a single point which is an end of either $\perp_{u}$ or $\perp_{v}$.

Theorem 4 ([10]). Every plane triangulation has a $\perp$-contact representation such that each inner face is represented by a rectangular region.

Following the ideas of de Fraysseix et al. [10], the $\perp$-contact representation $\mathcal{C}$ can be constructed as described in detail by Alam et al. [3, Section 3.1]. Observe that the $\perp$-shapes of three outer vertices may be pruned to segments such that the outer face is the complement of a rectangle. Moreover, note that every horizontal segment has a unique vertical segment touching it from above. The segments of the $\perp$-shapes of inner vertices partition the outer rectangle into finitely many rectangles; such a partition is called a rectangular layout. By Theorem $4, \mathcal{C}$ yields a rectangular layout $\mathcal{L}$ in which every rectangle $r$ corresponds to a face $f_{r}$ of $G$. In a generic rectangular layout no four rectangles meet in one point. In this case, we may define a segment of $\mathcal{L}$ as a maximal segment belonging to the boundary of the rectangles. Indeed, $\mathcal{C}$ can be constructed such that all horizontal and vertical segments of $\mathcal{C}$ have their private supporting line. Therefore, the segments of $\mathcal{C}$ are segments of $\mathcal{L}$ and are interiorly disjoint.

In the second step, we want to achieve correct areas in a weakly equivalent layout. Two rectangular layouts are weakly equivalent if there exist bijections between their horizontal and vertical segments, respectively, which preserve the ends on-relation, i.e., a segment $s$ ends on a segment $t$ in one layout if and only if the same holds for their images in the other layout. We make use of the following theorem.

Theorem 5 ([12]). For every rectangular layout with area assignment $\mathfrak{a}$ on the inner rectangles, there exists a weakly equivalent rectangular layout realizing the areas of $\mathfrak{a}$.

For this theorem several variants and proofs are known; we refer to [12, 14, 28]. For each rectangle $r$ corresponding to the face $f_{r}$, we set $\mathfrak{a}(r):=\mathcal{A}\left(f_{r}\right)$. By Theorem 5 , we obtain a weakly equivalent rectangular layout $\mathcal{L}^{\prime}$ in which the area of each rectangle $r$ is $\mathfrak{a}(r)$. Due to the weak equivalence of $\mathcal{L}$ and $\mathcal{L}^{\prime}$, the layout $\mathcal{L}^{\prime}$ can be viewed as a $\perp$-contact representation $\mathcal{C}^{\prime}$, which now realizes the areas. The weakly equivalent layout $\mathcal{L}^{\prime}$ is unique and may have four rectangles meeting in a point. This corresponds to the fact that two segments of $\mathcal{L}$ both end in the same point of a segment in $\mathcal{L}^{\prime}$ from different sides. For our further construction, we consider these two segments as two segments of $\mathcal{L}^{\prime}$.

In the third step, we obtain a 1-bend drawing of $G$. We define the following, usually degenerate, drawing $D_{\perp}$ with the help of $\mathcal{C}^{\prime}$ :

- Place each vertex $v$ in the heart of $\perp_{v}$; for a pruned $\perp$-shape, the heart is the bottom or left endpoint of the segment. 
- An edge $e=(u, v)$ is supported by the segments of $C^{\prime}$ in the following way: Since the two vertices $u$ and $v$ share an edge, $\perp_{u}$ and $\perp_{v}$ have a point of contact $\mathrm{c}$ in which a vertical and a horizontal segment meet. We draw the edge $e$ from the heart of $\perp_{u}$ along a segment of $\perp_{u}$ to the contact point $c$ and then along a segment of $\perp_{v}$ to the heart of $\perp_{v}$, see Figure 7. Note that $e$ has a unique bend point $c$.

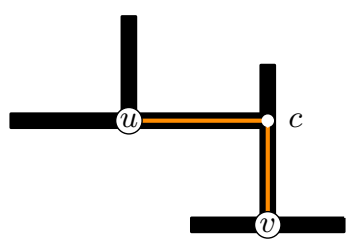

Figure 7: Definition of $D_{\perp}$.

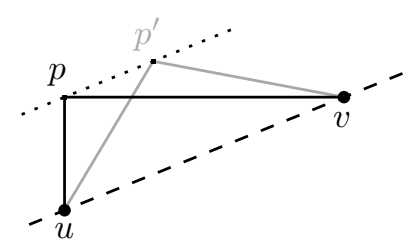

Figure 8: A parallel shift from $p$ to $p^{\prime}$.

Note that two edges may intersect along segments but never form a proper crossing. Hence the properties of a degenerate drawing of $G$ are fulfilled. By construction, each edge consists of a horizontal and a vertical segment and, hence, has at most one bend.

- Observation 8. The drawing $D_{\perp}$ is a degenerate 1-bend drawing of $G$ realizing $\mathcal{A}$.

For the fourth step, it remains to remove the degeneracies of $D_{\perp}$. For an edge $e$, we interpret its bend point $p_{e}$ as a vertex of degree 2 , and refer to the two incident edges of $p_{e}$ as its horizontal and vertical segments. As part of the degeneracies, bend points intersect non-incident edges. We handle this issue by parallel shifts. A parallel shift of a bend point $p$ in a drawing $D$ yields a (degenerate) drawing $D^{\prime}$ of $G$ in which only the position of $p$ and its edges changed: Let $p$ and $p^{\prime}$ denote the positions of the bend in $D$ and $D^{\prime}$, respectively, and let $u$ and $v$ denote its the two neighbors. A (degenerate) drawing $D^{\prime}$ of $D$ is obtained by a parallel shift of $p$ if the segment $p p^{\prime}$ is parallel to the segment $u v$, see Figure 8. A bend point $p$ is shiftable in $D$ if there exists a parallel shift of $p$. For instance, the topmost blue bend in Figure 9 (a) is not shiftable since every shift of it results in a proper crossing.

- Observation 9. A parallel shift of a bend point keeps all face areas invariant.

The contact representation $\mathcal{C}^{\prime}$ induces a coloring and an orientation of the inner edges: each edge corresponds to a contact point of two $\perp$-shapes. Orient the edge such that it is an outgoing edge for the vertex belonging to $\perp$-shape whose end is the contact point. Color the edge red, blue, or green, depending on the type of end which belongs to the contact point: Red for the top end, blue for the left end, and green for the right end of the $\perp$-shape. (Such a coloring and orientation is called a Schnyder wood of $G$.)

We analyze the structure of the neighborhood for a vertex $v$ in $D_{\perp}$, see Figure 9 (a). By construction, $v$ has three outgoing edges such that all incoming edges partially run on one of these outgoing edges. As noted before, in $C^{\prime}$ every horizontal segment has a unique vertical segment touching it from above and the point of contact corresponds to the heart of the $\perp$-shape. By definition of $D_{\perp}$, a vertex is placed in this contact point. Thus, in $D_{\perp}$ every horizontal segment has no segment touching it from above. Therefore, the bend point of the lowest green and lowest blue incoming edges is shiftable upwards. Due to the fact that every rectangle has positive area, some space is guaranteed. Therefore, we can parallel 


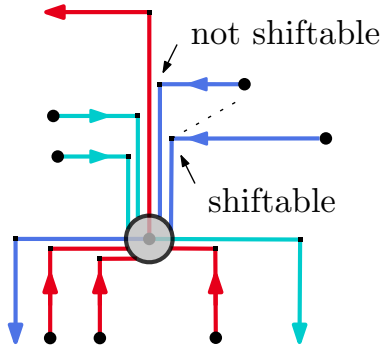

(a) The structure of the neighborhood of a vertex in the degenerate drawing $D_{\perp}$.

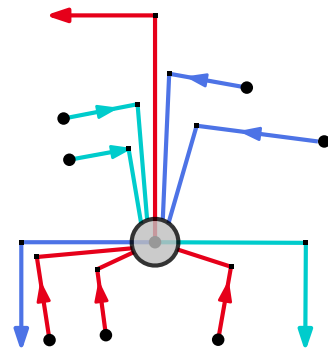

(b) The structure of the neighborhood of a vertex in the final 1-bend drawing.

Figure 9: The structure of the neighborhood of a vertex in $D_{\perp}$ and in the final drawing.

shift the bend point such that its edges are free of degeneracies. In particular, the bend point does not intersect non-incident edges anymore. Consequently, the bend point of the second lowest incoming edge of $v$ becomes shiftable. We iterate this process for all vertices such that all blue and green bend points are free of degeneracies. Afterwards, only red bend points are involved in degeneracies.

For every vertex, we consider the incoming red edges, which have either a left or a right bend with respect to the orientation. Note that there may be a unique red incoming edge for each vertex without a bend. Consider the rightmost right bend point (and likewise the leftmost left bend point). Its horizontal segment has no segment touching it from below since it is rightmost (leftmost) and the vertical segment is free of degeneracies, since by the first step there is no green or blue bend point on a red segment. Consequently, the rightmost right (leftmost left) bend point is shiftable to the bottom. We shift it parallel downwards such that no new degeneracies are introduced. Hence, the number of degeneracies decreased. Moreover, this process achieved that the second rightmost (leftmost) bend point becomes shiftable. By iterating, we remove all degeneracies. Finally, we obtain a 1-bend drawing $D_{\perp}^{*}$ of $G$ which is free of degeneracies and realizes $\mathcal{A}$.

\subsection{Bounds on the bend number}

Knowing that one bend per edge is always sufficient, we wonder how many bends may be necessary to realize an area assignment of a plane graph? We define the bend number of a plane graph $G$, denoted by $b(G)$, as the minimum number of bends which is always sufficient and sometimes necessary to realize any given area assignment of $G$.

Alternatively, Theorem 3 establishes the fact for every plane graph, there exists an area-universal subdivision. The subdivision number of a plane graph $G$, denoted by $s(G)$, is the minimum number of subdivision vertices which need to be inserted such that the subdivision of $G$ is area-universal. If $G$ is area-universal, then $s(G)=0$. Clearly, the bend number is upper bounded by the subdivision number. However, equality of both parameters is not clear since for the bend number the bended edges can be chosen with respect to the area assignment whereas for the subdivision number the bended edges must coincide for all area assignments. Theorem 3 assures the following proposition. 
- Proposition 10. For every plane graph $G=(V, E)$ it holds that $0 \leq b(G) \leq s(G) \leq|E|$.

We construct a family of graphs with the octahedron graph showing that the bend number, and thus the subdivision number, may be linear in the number of edges.

- Lemma 11. For every $k \geq 8$, there is a plane triangulation $G_{k}=(V, E)$ on $n:=4 k-6$ vertices and an area assignment $\mathcal{A}$, such that in every $\mathcal{A}$-realizing polyline drawing of $G_{k}$ at least $1 / 12$ of the edges have a bend, i.e., $b\left(G_{k}\right) \geq 1 / 12|E|$.

Proof. Recall that, by Theorem 1, the octahedron graph is not area-universal. Hence, there exists an area assignment $\mathcal{A}_{0}$ which has no realizing straight-line drawing. Thus in every $\mathcal{A}_{0}$-realizing polyline drawing of the octahedron at least one of twelve edges has a bend.

Now, we construct a plane triangulation $G_{k}$ for each $k \geq 8$. For $k \in\{3,6\}$, and $k \geq 8$, there exists an Eulerian triangulation on $k$ vertices. ${ }^{2}$ Therefore, we take an Eulerian triangulation $T$ on $k$ vertices and consider an inner face 2-coloring with $(k-2)$ white faces. We insert a copy of the plane octahedron graph $H$ into each white face $f$ of $T$ in the following way: identify three outer vertices of $H$ with the vertices of $f$. This yields a graph $G_{k}$ on $k+3(k-2)=n$ vertices, consisting of $(k-2)$ octahedron graphs which are pairwise edgedisjoint. Let $\mathcal{A}$ be an area assignment such that the restriction to any copy of $H$ results in $\mathcal{A}_{0}$ and the remaining black faces of $T$ obtain some arbitrary value.

In an $\mathcal{A}$-realizing polyline drawing $D$ of $G_{k}$, each copy of $H$ has at least one bended edge, since $\mathcal{A}_{0}$ is not realizable by a straight-line drawing. By the edge-disjointness, every bended edge may satisfy at most one copy of a octahedron. Consequently, at least $k-2$ of the $12(k-2)$ edges have a bend. This implies the claim.

- Corollary 12. Let $\mathcal{G}_{m}$ be the set of plane graphs with $m$ edges. For the maximum bend and subdivision number of $\mathcal{G}_{m}$ it holds that:

$$
\lfloor 1 / 12 \cdot m\rfloor \leq \max _{G \in \mathcal{G}_{m}}\{b(G)\} \leq \max _{G \in \mathcal{G}_{m}}\{s(G)\} \leq m .
$$

We note that the octahedron graph may not yield a better lower bound.

- Proposition 13. The plane octahedron graph has bend number and subdivision number 1.

Proof. Let $G$ denote the plane octahedron graph. Since, by Theorem 1, $G$ is not areauniversal, it clearly holds that $1 \leq b(G) \leq s(G)$.

In the remainder we show that $s(G) \leq 1$. The proof consists of two ideas. Firstly, let $e$ be an edge of the triangle of inner vertices, see Figure 10. Deleting $e$ in $G$ gives a 4 -face $q$. Replacing $e$ by the other diagonal edge $\bar{e}$ of $q$ yields a plane 3 -tree $G^{\prime}$. Since plane 3 -trees are area-universal [8], we can find a realizing drawing for every area assignment of $G^{\prime}$. In particular, for a given area assignment $\mathcal{A}$ of $G$, we may consider an $\mathcal{A}^{\prime}$-realizing drawing $D^{\prime}$ of $G^{\prime}$ where $\mathcal{A}^{\prime}$ conforms to $\mathcal{A}$ except to the fact that the two faces of $q$ in $G^{\prime}$ obtain the sum of the prescribed areas of the two faces of $q$ in $G$.

\footnotetext{
${ }^{2}$ The existence of such an Eulerian triangulation follows from the following observation: Applying the $P$-rule defined by Batagelj [5] to an Eulerian triangulation on $k$ vertices results in an Eulerian triangulation on $k+2$ vertices. Moreover, there exists an Eulerian triangulation on six vertices (the octahedron) and on nine vertices (e.g., identify the outer face of a copy of the octahedron with an inner face of the octahedron).
} 


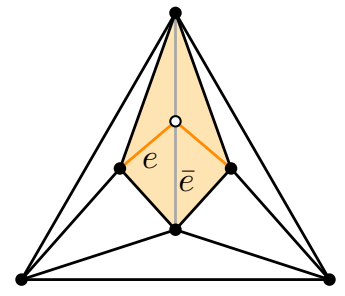

Figure 10: This subdivision of the octahedron graph is area-universal.

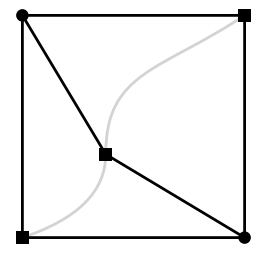

Figure 11: The plane quadrangulation $Q_{5}$ is strongly area-universal.

Secondly, the intermediate value theorem asserts a position for a bend point of $e$ on $\bar{e}$ in $D^{\prime}$ such that the area of $q$ is arbitrarily split among the two adjacent faces of $e$. Hence, each area assignment of $G$ is realizable with a bend on $e$.

A similar argument shows that the unique plane quadrangulation on five vertices, depicted in Figure 11, is area-universal in a strong sense. For simplicity, we denote it by $Q_{5}$. We say a graph is strongly area-universal if for every area assignment $\mathcal{A}$ there exists an $\mathcal{A}$-realizing drawing within every outer face of area $\Sigma \mathcal{A}$.

- Lemma 14. The plane quadrangulation $Q_{5}$ is strongly area-universal.

Proof. Let $\mathcal{A}$ be an area assignment of $Q_{5}$. For a prescribed outer face $O$ of area $\Sigma A$, we consider a curve $\mathcal{C}$ within $O$ connecting the two non-neighbors of the inner vertex $v$. Moving $v$ along $\mathcal{C}$, the area of the two inner faces changes continuously. Therefore, the intermediate value theorem guarantees a position of $v$ which yields an $\mathcal{A}$-realizing drawing. Note that it is crucial that the outer face of $Q_{5}$ is a 4 -face.

This yields the following fact.

- Proposition 15. Every 2-degenerate quadrangulation is strongly area-universal.

Proof. Any 2-degenerate quadrangulation can be constructed from a plane 4-cycle by iteratively inserting a vertex of degree 2 (acting as a subdivided diagonal) into an inner 4-face. In other words, in every step we choose an inner 4 -face $f$ of a quadrangulation and insert a copy of $Q_{5}$ by identifying the boundaries of $f$ and of the outerface of $Q_{5}$. Thus, Lemma 14 implies the claim.

Indeed, it may be true that plane bipartite graphs are area-universal. If so, this has interesting consequences for the subdivision number of plane triangulations.

- Proposition 16. If plane bipartite graphs are area-universal, then for every plane triangulation $T=(V, E)$ it holds that $b(T) \leq s(T) \leq 1 / 3|E|$.

Proof. Recall that a plane triangulation $T$ on $n$ vertices has $2 n-4$ faces (including the outer face). The dual graph $T^{*}$ of $T$ is a bridgeless cubic graph and hence, by Petersen's theorem, has a perfect matching $M^{*}$ of size $n-2$. Deleting the dual edges $M$ of $M^{*}$ in $T$ yields a quadrangulation $Q$. If plane bipartite graphs are area-universal, then there exists a realizing straight-line drawing for every area assignment of $Q$. By Lemma 14, we may reinsert the deleted edges of $M$ with one bend each. Therefore, subdividing the edges of $M$ in $T$ yields an area-universal subdivision where $n-2$ of the $3(n-2)$ edges are subdivided. 


\subsection{Fewer Bends for Plane Bipartite Graphs}

Now, we discuss the subdivision number of plane bipartite graphs. Interestingly, no plane bipartite graph is known that needs a bend. Besides Proposition 15, Evans et al. [13] show that (square) grid graphs are area-universal. Otherwise, area-universality of plane bipartite graphs is wide open. In this section, we improve the upper bound on the number of bends that always suffice for plane bipartite graphs. We show that at most half of the edges need a bend. Indeed, we can place the bends on the same edges independent of the area assignment. Hence, we obtain a bound on the subdivision number.

Theorem 6. Let $G=(X \cup Y, E)$ be a plane bipartite graph. Then, $s(G) \leq 1 / 2|E|$.

Proof. First, we consider the case that $G$ is a quadrangulation and show that an arbitrary but fix area assignment $\mathcal{A}$ has an $\mathcal{A}$-realizing 1-bend drawing of $G$ with at most $1 / 2|E|$ bends. Moreover, we place the bends on the same edges independent of $\mathcal{A}$. The proof consists of four steps, illustrated in Figure 12. The main difference to the proof of Theorem 3 lies in Step 1. Moreover, Steps 3 and 4 are a little more intricate. The procedure is as follows:

0. (Augment $G$ to a quadrangulation.)

1. Take a segment contact representation $\mathcal{C}$ yielding a rectangular layout $\mathcal{L}$.

2. Obtain a weak equivalent rectangular layout $\mathcal{L}^{\prime}$ realizing the areas.

3. Define a degenerate drawing $D$ on $\mathcal{L}^{\prime}$.

4. Construct a non-degenerate drawing $D^{*}$ from $D$ by parallel shifts.

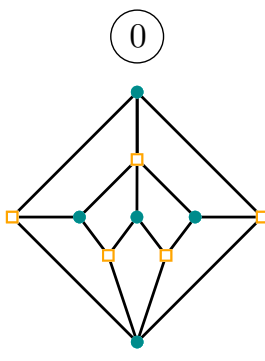

$G$

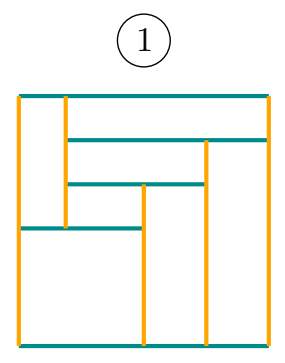

$\mathcal{C}$

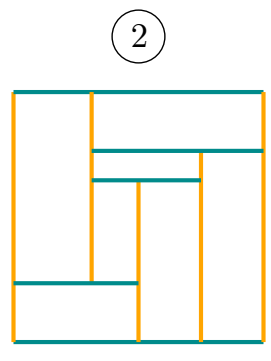

$\mathcal{L}^{\prime}$

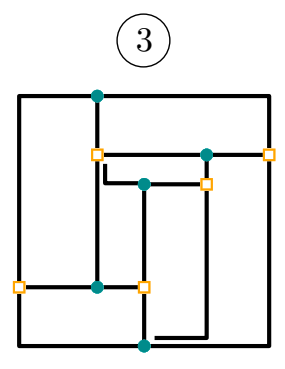

$D$
(4)

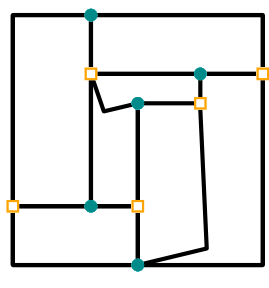

$D^{*}$

Figure 12: Construction of a 1-bend drawing realizing the prescribed areas in four steps.

In the first step, we take a segment contact representation of $G=(X \cup Y, E)$. This is a family $\left\{s_{v} \mid v \in X \cup Y\right\}$ of horizontal and vertical segments where $s_{v}$ and $s_{u}$ intersect if and only if $(u, v) \in E$. Moreover, each non-empty intersection consists of a single point which is an endpoint of at least one of the segments.

Theorem 7 ([9, 15]). Every plane quadrangulation has a segment contact representation such that each inner face is represented by a rectangle.

Let $\mathcal{C}$ be a segment contact representation of $G$. We assume that the vertices of $X$ are represented by vertical segments. The segments of $\mathcal{C}$ partition a rectangle into rectangles, and hence, $\mathcal{C}$ yields a rectangular layout $\mathcal{L}$. In the second step, we obtain a weakly equivalent rectangular layout $\mathcal{L}^{\prime}$ realizing the areas by Theorem 5; let $\mathcal{C}^{\prime}$ denote the corresponding 
segment contact representation. In the third step, we define a degenerate drawing $D$ from $\mathcal{C}^{\prime}$. The challenge is to place the vertices such that, firstly, we insert the bends on the same edges, secondly, we save one bend per vertex, and thirdly, we can remove the degeneracies by parallel shifts. We distinguish two cases depending on the minimal degree $\delta$ of $G$. Note that in a quadrangulation $\delta$ is 2 or 3 .

Case 1: $\delta(G)=3$. Since every segment has only two endpoints but at least three contacts, every segment has an inner contact point. For a vertical segment $s_{v}$, an inner contact point is right if it is the endpoint of a horizontal segment touching $s_{v}$ from the right. Otherwise, it is left. Likewise, we distinguish inner top and bottom contact points of horizontal segments. We construct $D$ from $\mathcal{C}^{\prime}$ as follows:

- A vertex $v \in X$ is placed on topmost right inner contact point of the segment $s_{v}$ if it exists; otherwise $v$ is placed on the topmost left inner contact point.

- A vertex $v \in Y$ is placed on leftmost bottom inner contact point of the segment $s_{v}$ if it exits; otherwise $v$ is placed on the leftmost top inner contact point.

- An edge $e=(v, w) \in E$ is drawn from $v$ along $s_{v}$ to the contact point of $s_{v}$ and $s_{w}$, and then along $s_{w}$ to $w$.

Note that with this definition the vertices are placed on the same contact points independent of the area assignment. This allows us to obtain an area-universal subdivision of $G$. By placing a vertex on an inner contact point of its segment, the corresponding edge has no bend. Hence, we save one bend per vertex and the number of bends is at most $|E|-|V|$.

- Observation 17. The drawing $D$ is a degenerate 1-bend drawing of $G$ realizing $\mathcal{A}$ with at most $|E|-|V|$ bends.

In the fourth step, we remove the degeneracies by parallel shifts of bend points. By the placement of the vertices, a vertical segment $s_{v}$ has no right bend point above $v$ and a horizontal segment $s_{v}$ has no bottom bend point left of $v$. This property is our invariant $4_{0}$. For a vertex $v \in X(v \in Y)$ with a vertical (horizontal) segment $s_{v}$, we partition the inner bend points into three sets: $\mathcal{B}_{1}\left(s_{v}\right)$, the set of right (bottom) bend points on $s_{v}, \mathcal{B}_{2}\left(s_{v}\right)$, the set of left (top) bend points on $s_{v}$ which are below (right of) $v$, and $\mathcal{B}_{3}\left(s_{v}\right)$, the set of remaining bend points, i.e., left (top) bend points on $s_{v}$ which are above (left of) $v$, see also Figure 13 (a). For a segment $s_{v}$ and all $i \in\{1,2,3\}$, a bend point $b \in B_{i}(s)$ is smaller than $b^{\prime} \in B_{i}(s)$ if $b$ is closer to $v$ in $D$ than $b^{\prime}$.

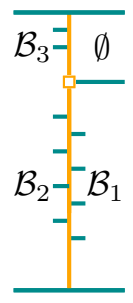

(a) Definition of $D$ and $\mathcal{B}_{i}$.

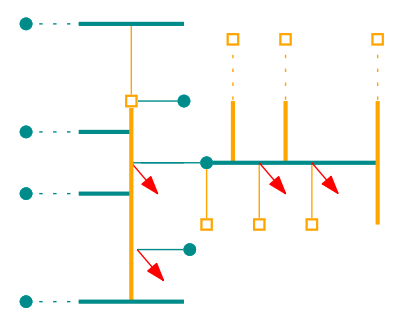

(b) Illustration of Step $4_{1}$.

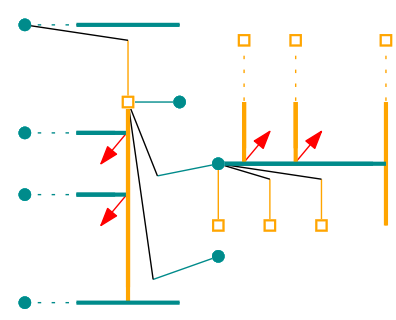

(c) Illustration of Step $4_{2}$.

Figure 13: Illustration of Step 3 and Step 4 in Case 1 of the proof of Theorem 6. 
We remove the degeneracies in three steps. For $i \in[3]$, Step $4_{i}$ is defined as follows:

Step $4_{i}$ : For each $v \in X \cup Y$, do: while $\mathcal{B}_{i}\left(s_{v}\right)$ is not empty, choose the smallest $b \in \mathcal{B}_{i}\left(s_{v}\right)$, parallel shift $b$, and delete $b$ from $\mathcal{B}_{i}\left(s_{v}\right)$.

If Step $4_{i}$ is completed successfully, then invariant $4_{i}$ holds, namely that every segment has no bend points in $B_{j}\left(s_{v}\right)$ for $0 \leq j \leq i$. Indeed invariant $4_{i}$ is necessary in order to perform Step $4_{i+1}$. Clearly, invariant $4_{3}$ guarantees a non-degenerate 1-bend drawing of $G$ which realizes $\mathcal{A}$ and has at most $|E|-|V|$ bends. Hence it remains to show that the smallest $b \in \mathcal{B}_{i}\left(s_{v}\right)$ is shiftable if invariant $4_{i-1}$ holds.

Consider a vertex $v \in X$ with vertical segment $s_{v}$ and the smallest $b \in \mathcal{B}_{1}\left(s_{v}\right)$, see Figure $13(\mathrm{~b})$. By invariant $4_{0}$, the horizontal edge of $b$ is free of bottom contact points. Since $b$ is the smallest in $\mathcal{B}_{1}\left(s_{v}\right)$, the vertical edge of $b$ is free of right contact points. Therefore, $b$ is shiftable bottom-rightwards. Moreover, after shifting and deleting $b$, the new smallest bend point becomes shiftable. The argument for $v \in Y$ is analogous. More generally, for $i \in\{1,2,3\}$, the smallest bend point $b \in \mathcal{B}_{i}\left(s_{v}\right)$ is shiftable since both of its edge are free of bend points to one side; for one edge this is guaranteed by the invariant $4_{i-1}$, for the other edge by the fact that $b$ is the smallest bend point in $b \in \mathcal{B}_{i}\left(s_{v}\right)$.

Case 2: $\delta(G)=2$. If it exists, choose an inner vertex of degree 2 and remove the segment $s_{v}$ in $\mathcal{C}^{\prime}$. This results in a quadrangulation where two faces are unified to one new face. Assign the sum of the two face areas to the new face. Delete inner vertices of degree 2 until all inner vertices are of degree at least 3 . This yields a graph $G^{\prime}$ with area assignment $\mathcal{A}^{\prime}$. Proceed with $G^{\prime}$ as in Case 1 with some extra care. If one or several outer vertices have degree 2 , we place them on distinct ends of their segments; for instance in clockwise order. Moreover, we make the parallel shifts small enough, such that the following special property is fulfilled in an $\mathcal{A}^{\prime}$-realizing drawing of $G^{\prime}$ : up to a tiny $\varepsilon$ with $n \varepsilon \ll A_{\text {min }}$, each face $f$ of $G^{\prime}$ contains an axis-aligned rectangle with area $\mathcal{A}^{\prime}(f)-\varepsilon$, where $A_{\text {min }}:=\min _{f \in F^{\prime}(G)} \mathcal{A}(f)$.

We use the special property to reinsert the degree 2 vertices in reverse order of deletion and obtain a sequence of drawings $\left(G_{k}^{\prime}\right)_{k}$. We use the invariant that $G_{k}^{\prime}$ is a nondegenerate drawing where each face area is realized by an axis-aligned rectangle up to $k \varepsilon$. Consider the $(k+1)^{\text {th }}$ vertex $v$ of degree 2 and the face $f$ in $G_{k}$ where $v$ must be inserted. Assume $f$ has area $a_{1}+a_{2}$ and must be split into two faces $f_{1}$ and $f_{2}$ of area $a_{1}$ and $a_{2}$. By assumption, $a_{1}, a_{2} \geq A_{\min }$ and $f$ contains an axis-aligned rectangle $R$ of area $a_{1}+a_{2}-k \varepsilon$, see Figure 14. Assume that $v \in X$. By the intermediate value theorem, there exists a vertical segment $s$ within $R$ such that $s$ dissects $f$ into two parts of area $a_{1}$ and $a_{2}$, respectively. Place $v$ on one endpoint of $s$ and a bend point $b$ on the other endpoint of $s$. Note that the areas of $f_{1}$ and $f_{2}$ are realized by a rectangle up to $k \varepsilon$. In order to remove the degeneracies,

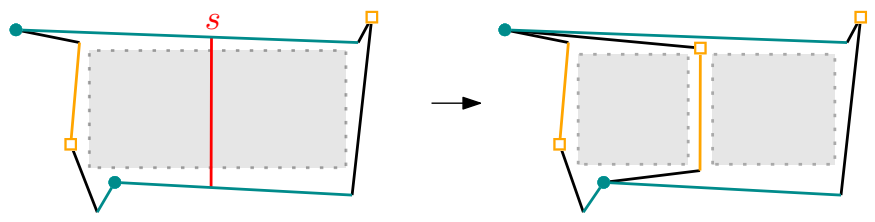

Figure 14: Insertion of an inner vertex of degree 2 in Case 2. 
use parallel shifts of $v$ and $b$ which are small enough to guarantee that $f_{i}$ contains a rectangle of area $a_{i}-(k+1) \varepsilon$. This ends our treatment of Case 2 .

If $G$ is not a quadrangulation, then we consider a quadrangulation $Q$ with $G$ as an induced subgraph. For each face in $G$, we partition its area assignment among its subfaces in $Q$ and obtain $\mathcal{A}^{\prime}$. Clearly, an $\mathcal{A}^{\prime}$-realizing 1-bend drawing of $Q$ induces an $\mathcal{A}$-realizing 1-bend drawing of $G$. However, the number of bends may exceed $1 / 2|E|$. Therefore, we ensure to save one bend per vertex by placing the vertices on inner contact points which belong to edges of $G$. To do so, delete all segments belonging to artificial vertices in $\mathcal{C}$. If necessary, remove vertices of low degree iteratively as in Case 2. Afterwards, place vertices, remove degeneracies and reinsert vertices of $G$ with low degree as in Case 1 and Case 2 . Note that degree 1 vertices may also appear, but can easily be reinserted.

A planar bipartite graph has at most $2|V|-4$ edges. Therefore, the number of edges with bends is at most $|E|-|V| \leq|V|-4$, and the number of edges without bends is at least $|V|$. Consequently, in all cases the number of bends is less than $1 / 2|E|$.

If in the third step of the last proof, the vertices are placed in fortunate positions, one can save additional bends. In particular, every corner of the outer rectangle has two closest contact points, one on each incident segment; if in both of these a vertex is placed, then one bend can be saved. For the cube graph this allows to save four bends and shows that the cube graph as a planar partial 3-tree is not only area-universal but also convex area-universal, i.e., every area assignment has a realizing drawing with convex faces.

- Proposition 18. The cube graph is convex area-universal.

Proof. Let $\mathcal{A}$ be an area assignment of the cube graph. Then $\mathcal{A}^{\prime}$ denotes the area assignment where the areas of the four boundary faces are doubled with respect to $\mathcal{A}$. Theorem 6 guarantees the existence of a 1-bend drawing realizing $\mathcal{A}^{\prime}$. Indeed, already the drawing $D$ defined in the third step of the proof of Theorem 6 is free of degeneracies. Moreover, as illustrated in Figure 15 (c), every outer edge has exactly one bend and all inner edges are segments. Replacing the outer edges by segments halves the area of the boundary faces and consequently gives an $\mathcal{A}$-realizing straight-line drawing with convex faces.

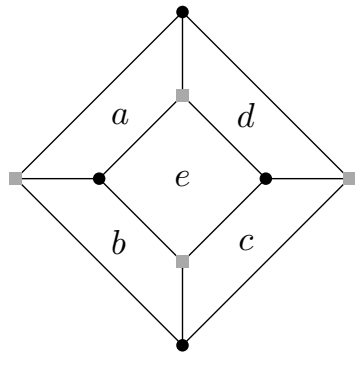

(a) The cube graph with an area assignment $\mathcal{A}$.

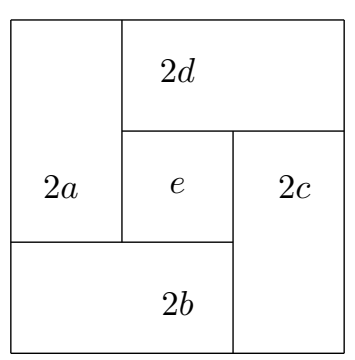

(b) A rectangular layout realizing $\mathcal{A}^{\prime}$.

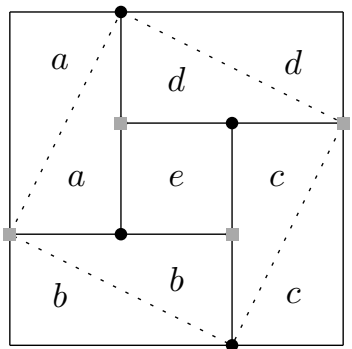

(c) A convex straight-line drawing realizing $\mathcal{A}$.

Figure 15: Illustration of the proof of Proposition 18. 


\section{Further research directions}

Various interesting questions remain open. We comment on three directions.

Area-universal graph classes A very promising candidate for a further area-universal graph class are the plane bipartite graphs. In the conference version of this article [18], we asked if 3-connected quadrangulations are even convex area-universal. In the meantime, we were able to answer this question in the negative. A counter example can be found in my $\mathrm{PhD}$ thesis [19] - together with some first tools to tackle the open problem. Further partial results in this direction are the area-universality of 2-degenerate quadrangulations as discussed in Proposition 15 and the fact that grid graphs are area-universal [13].

Computational Complexity As mentioned in the introduction, Dobbins et al. [11, 20] conjecture that the computational complexity of deciding whether a plane graph is areauniversal is $\forall \exists \mathbb{R}$-complete. The complexity class $\forall \exists \mathbb{R}$ is a natural generalization of $\exists \mathbb{R}$, a complexity class capturing the computational complexity of many interesting geometric problems. It lies somewhere between NP and PSPACE:

$$
\mathrm{NP} \subseteq \exists \mathbb{R} \subseteq \forall \exists \mathbb{R} \subseteq \text { PSPACE }
$$

Area-universality may turn out to be the first natural geometric problem that is complete for this class. This would give the complexity class $\forall \exists \mathbb{R}$ some relevance.

Optimal Bend Drawings Theorem 3 establishes the fact that one bend per edge always suffices to realize any given area assignment for every plane graph. But how many bends are really necessary? It seems that the number of necessary bends may be a lot smaller. In Section 3, we introduced the bend number and subdivision number of a plane graph as possible notions for optimal bend drawings. Recall that, by Proposition 10, for every plane graph $G=(V, E)$, it holds that

$$
0 \leq b(G) \leq s(G) \leq|E|
$$

For optimal bend drawings, one can start with the following questions:

- What are the bend and subdivision numbers of the icosahedron graph?

- What is the maximum subdivision number of a plane graph with $m$ edges?

(Lemma 11 and Theorem 3 show that the number lies between $\lfloor m / 12\rfloor$ and $m$.)

- Do there exist graphs for which the bend number and the subdivision number differ? Is the subdivision number bounded by a function of the bend number?

Acknowledgements. I thank Stefan Felsner, Udo Hoffmann, and Daniel Schmidt genannt Waldschmidt for discussions about the problem and helpful comments on drafts of this manuscript. Moreover, I would like to thank the anynomous reviewers for valuable suggestions that helped to improve the presentation of this paper. 


\section{References}

[1] graphdrawing.org. http://www.graphdrawing.org/.

[2] Muhammad Jawaherul Alam, Therese C. Biedl, Stefan Felsner, Michael Kaufmann, and Stephen G. Kobourov. Proportional contact representations of planar graphs. Journal of Graph Algorithms and Applications, 16(3):701-728, 2012.

[3] Muhammad Jawaherul Alam, Therese C. Biedl, Stefan Felsner, Michael Kaufmann, Stephen G. Kobourov, and Torsten Ueckerdt. Computing cartograms with optimal complexity. Discrete \& Computational Geometry, 50(3):784-810, 2013.

[4] Anders Bager. Lösung der Aufgabe 260. Elemente der Mathematik, 12:43, 1957.

[5] Vladimir Batagelj. An improved inductive definition of two restricted classes of triangulations of the plane. Banach Center Publications, 25(1):11-18, 1989.

[6] Giuseppe Di Battista, Peter Eades, Roberto Tamassia, and Ioannis G. Tollis. Graph Drawing: Algorithms for the Visualization of Graphs. Prentice Hall, Upper Saddle River, NJ, 1998.

[7] Anna Bernáthová. Kreslení grafu s podmínkami na velikosti sten. Bachelor thesis, Charles University Prague, 2009. Available at https://is.cuni.cz/webapps/zzp/ detail/63479/20281489/.

[8] Therese C. Biedl and Lesvia Elena Ruiz Velázquez. Drawing planar 3-trees with given face areas. Computational Geometry, 46(3):276-285, 2013.

[9] Hubert de Fraysseix, Patrice Ossona de Mendez, and János Pach. Representation of planar graphs by segments. Intuitive Geometry, 63:109-117, 1991.

[10] Hubert de Fraysseix, Patrice Ossona de Mendez, and Pierre Rosenstiehl. On triangle contact graphs. Combinatorics, Probability and Computing, 3:233-246, 1994.

[11] Michael G. Dobbins, Linda Kleist, Tillmann Miltzow, and Paweł Rzążewski. Is area universality $\forall \exists \mathbb{R}$-complete? In Andreas Brandstädt, Ekkehard Köhler, and Klaus Meer, editors, Proc. Int. Graph-Theoretic Concepts in Computer Science (WG 2018), LNCS, Berlin, Heidelberg, 2018. Springer. accepted, preprint ArXiv:1712.05142.

[12] David Eppstein, Elena Mumford, Bettina Speckmann, and Kevin Verbeek. Areauniversal and constrained rectangular layouts. SIAM Journal on Computing, 41(3):537$564,2012$.

[13] William Evans, Stefan Felsner, Michael Kaufmann, Stephen G. Kobourov, Debajyoti Mondal, Rahnuma Islam Nishat, and Kevin Verbeek. Table cartogram. Computational Geometry, 68:174-185, 2017. Special Issue in Memory of Ferran Hurtado.

[14] Stefan Felsner. Exploiting air-pressure to map floorplans on point sets. Journal of Graph Algorithms and Applications, 18(2):233-252, 2014. 
[15] Irith Ben-Arroyo Hartman, Ilan Newman, and Ran Ziv. On grid intersection graphs. Discrete Mathematics, 87(1):41-52, 1991.

[16] Henning Heinrich. Ansätze zur Entscheidung von Flächenuniversalität. Masterarbeit, Technische Universität Berlin, 2018. Available at www.math.tu-berlin.de/ felsner/ Diplomarbeiten/heinrich.pdf.

[17] Michael Kaufmann and Dorothea Wagner, editors. Drawing Graphs: Methods and Models, volume 2025 of LNCS. Springer, 2003.

[18] Linda Kleist. Drawing planar graphs with prescribed face areas. In Pinar Heggernes, editor, Proc. Int. Graph-Theoretic Concepts in Computer Science (WG 2016), volume 9941 of $L N C S$, pages 158-170, Berlin, Heidelberg, 2016. Springer.

[19] Linda Kleist. Planar Graphs and Face Areas - Area-Universality. PhD thesis, Technische Universität Berlin, 2018.

[20] Linda Kleist, Tillmann Miltzow, and Paweł Rzążewski. Is area universality $\forall \exists \mathbb{R}$ complete? In Proceedings of the 33th European Workshop on Computational Geometry (EuroCG 2017), pages 181-184, 2017.

[21] Takao Nishizeki and Md. Saidur Rahman. Planar Graph Drawing, volume 12 of Lecture Notes Series on Computing. World Scientific, 2004.

[22] L. Bruce Richmond and Nicholas C. Wormald. Random triangulations of the plane. European Journal of Combinatorics, 9(1):61-71, 1988.

[23] Gerhard Ringel. Equiareal graphs. In Rainer Bodendiek, editor, Contemporary Methods in Graph Theory, in honour of Prof. Dr. K. Wagner, pages 503-505. Wissenschaftsverlag, Mannheim, 1990.

[24] Esther Szekeres. Einfache Beweise zweier Dreieckssätze. Elemente der Mathematik, $22: 17-18,1967$.

[25] Roberto Tamassia, editor. Handbook of Graph Drawing and Visualization. CRC Press, Boca Raton, FL, 2013.

[26] Carsten Thomassen. Plane cubic graphs with prescribed face areas. Combinatorics, Probability \& Computing, 1(4):371-381, 1992.

[27] Mu-Tsun Tsai and Douglas B. West. A new proof of 3-colorability of Eulerian triangulations. Ars Mathematica Contemporanea, 4(1), 2011.

[28] Shmuel Wimer, Israel Koren, and Israel Cederbaum. Floorplans, planar graphs, and layouts. IEEE Transactions on Circuits and Systems, 35:267-278, 1988. 